\title{
RELEASE OF RADIOISOTOPES AND ACTIVATED MATERIALS FROM NUCLEAR INSTALLATIONS AND FACILITIES
}

\author{
P. F. Manfredi J. E. Millaud \\ Lawrence Berkeley National Laboratory \\ 1, Cyclotron Rd, Berkeley CA 94720
}

\begin{abstract}
This report discusses the problems of release of items from facilities and installations where radiation-based activities have been carried out. Several situations are reviewed and their release problems are discussed in detail. Particular attention is devoted to the assessment of the activity of the items to be released. A correct assessment of the activity will help the decision about the final use of the items removed from the radiation-related facility, either re-use, entering the public market, recycling, disposal and storage under different procedures. Even the final destination of the building which hosted the facility needs to be decided on the basis of an accurate assessment of the residual activity. The assessment of the activity, besides being fundamental in guaranteeing a safe approach to the procedures related to the release may result in a substantial profit. This is the case of items whose level of activity is so low that they can be put on the public market, reused or recycled for final product subject to very stringent radiation safety requirements. It will be shown that detector techniques play a fundamental role in the release process. In particular, the low-level counting techniques are fundamental in establishing whether or not the unrestrained release is feasible or not.
\end{abstract}




\section{RELEASE OF RADIOISOTOPES AND ACTIVATED MATERIALS}

\section{1-INTRODUCTION}

The detection of radioactive material in steel scrap shows many of the challenges associated with detecting radiation at or below natural background activities-a foreseeable requirement for the release standards (1)(2)(3)(4).

The secretary of the Department of Energy has mandated the suspension of unrestricted release of radioactive materials originating within the DOE complex. Such release was governed by DOE order 5400.5. It is expected that new guidelines will be issued soon.

Under consideration is the characterization, sorting and recycling of metals -at least within the DOE complex-. If this is economically viable it should save substantial costs and lessen the demand on the alternative long term radioactive materials repositories. Public acceptance of such repositories has been so far very limited.

This report discusses the problems associated with the release of items from installations where a substantial radiation-connected activity has taken place. The word item underlines the fact that the process of release in this report does not merely refer to the radioactive wastes or activated materials that need to be disposed of. The concept of release here refers to a much broader spectrum of situations. Release is the process by which any item from the facility is cleared for any further actions, which may include its transportation, its processing by various techniques, its recycling, its storage or even its appearance on the public market. Among the item to be released there might even be the building where the radiation-connected activities are taking place.

The process of release, if correctly managed may result in substantial cost savings or even provide financial gains. If poorly managed it may result in risk of exposure for humans, in environmental hazard and in enormous costs associated with the unavoidable decontamination activities. Fundamental in the process of release is the assessment of the level of activity of the item to be cleared for further actions. An accurate assessment of the activity of the item, either wastes produced during the normal activity of the installation, material that are activated, contaminated liquids and gases, the building itself, is of paramount importance for the following reasons:

1 - It determines the precautions that are to be taken in order to bring the exposure of the operators within safe limits.

2 - It defines the nature of the disposal process and the final storage destination.

3 - It suggests the type of further processing, like reuse or recycling and the nature of successive applications that the released item may suit.

4 - It may open the way to the so called unrestricted or exempt release

A broad variety of techniques can be used for qualitative and quantitative characterization of radioactive materials. They could be enhanced by an assertive development program.

In what follows particular attention will be devoted to the release situation that are of concern for the United States Department of Energy. However, for a thorough understanding of the nature of the release problems, the situations that may arise in a broader set of contexts will be considered. 


\section{2-RADIATION-CONNECTED ACTIVITIES}

This section reviews the types of facilities and installations where radiation is an essential component in their normal activities. This will help to understand what kinds of release problems arise in the different contexts.

Radiation from natural and artificial radioisotopes and particles and photon beams created by man made sources like particle accelerators and nuclear reactors intervenes in a large number of applications.

There are countless examples, to support this statement.

In medicine radioisotopes are employed both as tracers in the diagnosis making process and in the treatment of several pathological conditions. Radiation based treatment is based also on particle accelerators.

Several applications in material science employ treatment by radiation emitted by radioisotopes or particle accelerators.

Some types of nondestructive tests on materials and structures utilize radiation for the purpose of detecting defects by imaging techniques.

Scientific institutions employ for research activities a large variety of radioactive sources, particles accelerators and nuclear reactors.

Sterilization of food, medicine and dentistry tools and materials in its most advanced form is based on radiation. We're informed in these days that radiation is being considered as a powerful way of killing bacteria in bio-terrorism prevention.

Security is also to some extent radioactivity dependent, for techniques base upon gamma-rays and neutrons can be employed to detect dangerous items hidden in luggage, vehicles, railway cars.

Radioisotopes are employed in several industrial contexts. Thickness and level meters based upon the measurement of the radiation absorption have been in use for a long time. Radioisotope-based elimination of static electricity in paper mills, plastics and printing ink industries is also common. Items, like smoke detectors include low level radioactive sources. Even the jewelry industry employs radioactivity. Gems are irradiated by neutron in a reactor in order to make them assume attractive colors and their release and shipping must take into consideration the fact that they become active by induced radioactivity.

Radioactivity is an essential component in the electricity production based on nuclear power plants.

Strategic and defense-related activities have extensively employed radioactivity throughout the second part of the twentieth century. Plants designed to enrich fissile materials and weaponry developing industry and laboratories have been processing a large amount of radioactive materials and created radioactive materials by induced activity. The military industry has also utilized similar technique to produce the fissile materials that are employed to power the nuclear submarines.

Nuclear chemistry and facilities operating on large volumes of radioisotopes employ the so called hot cells , that is, laboratories_designed to process radioisotopes of extremely high activity for a large variety of applications. 


\section{3-THE RELEASE OF RADIOACTIVE ITEMS}

From the previous discussion it becomes clear that some of the discussed facilities employ radioactive materials as fundamental components of their every day's activity. Some other ones, like particle accelerators, for instance, may create active materials by induced radioactivity. Among the industrialized countries, the United States is beyond any doubt the one where radioactivity had the largest application impact. As stated by Bill Richardson, former Energy Secretary, with reference to DOE facilities,

"It si critical to our national security, health and safety, and environmental protection that we take proper care of our nuclear legacy. Currently (July 2000) more than half of the department's nuclear materials management facilities is over 40 years old. This plan lays the ground work for how we can address not only an aged infrastructure, but also the complex set of responsibilities related to managing and disposing a sizable and diverse nuclear materials inventory".

A fundamental aspect in the responsibilities connected with the nuclear legacy in the United States is the already defined release of radioactive items. Depending on the facility under consideration, the release may occur on a periodical basis or at the moment when the facility is decommissioned to be permanently closed. Whatever the nature of the radioactive item is, its clearance for release requires, as already pointed out, an accurate assessment of its activity. It must be ensured, indeed, that any release of radioactive material from the facility does not create to people and to the environment conditions of exposure beyond prescribed limits. Besides, provisions should be made in order to mitigate the effects in the unfortunate event that an accident related to the release of radioactive materials occurs.

The problems related to the release of radioactive materials are becoming more and more important as more and more facilities are decommissioned.

The release aspects are vastly different depending on the type of facility they come from. The next section will clarify the nature of the problem in the more common situations.

\section{4-THE NATURE OF THE RELEASE PROCESS IN DIFFERENT CONTEXTS}

\section{4-1 Release of radioactive items from medical facilities.}

The medical applications provide interesting examples of different types of radioactive material release. There is the continuous release of radionuclides employed in hospitals for diagnostic purposes. They are usually short-lived radioisotopes.

Radiotherapy by beams of particles and photons creates release problems of a different nature. The release of waste and materials occurs when the therapy units are decommissioned. The decommissioning of a ${ }^{60} \mathrm{Co}$ therapy unit, which normally employs a source of very high activity, up to hundreds of Curies, is a difficult process which requires highly specialized and specifically trained operators.

Therapy based on beams created by particle accelerators does not employ radioisotopes and is, to a large extent, free from contamination problems. However, the decommissioning of an accelerator usually brings about release problems related to the fact that during the operational life of the accelerator its beam inevitably activates materials. The problems of release of materials activated by an accelerator employed for 
therapy purposes are usually not very hard, because the induced activity can be assumed to be comparatively weak.

A problem which arises sometimes in hospitals, is the release, that is the clearance of rooms that were originally employed as radioisotope laboratories to a different use.

This is a particular case of the building release problem, which will be discussed in detail in the section devoted to the DOE specific release aspects.

\section{4-2 Release of radioactive items from the electricity generating industry}

The industry producing electricity by nuclear methods has a large variety of release problems that start from the fuel cycle, continue throughout the active life of the power generating plant and have a large impact when the power station needs to be decommissioned.

The nuclear fuel cycle generates some radioactive waste at each step. From the Uranium mining and fuel fabrication to the reprocessing of spent fuel, large quantities of wastes of different levels of activity are created. If the spent fuel is not reprocessed, it becomes itself a waste.

The operation of a nuclear power station implies a large variety of release situations that involve parts and components that need a periodical replacement, as well as contaminated liquids that need to be disposed of.

The decommissioning of a nuclear power station is an extremely complex procedure from the point of view of the release of wastes and materials. This is related to the very large amounts of materials of different levels of activity that are to be released. There are materials of low activity, like the rubble resulting from the demolition of walls and shields. However, there are also to very active components and parts in the reactor structure.

\section{4-3 Release of radioactive items from scientific institutions}

Scientific institutions also present a large variety of situations relevant to the release of radioactive materials and components. They include:

Release of radioisotopes that are no longer used. This include the case of small sealed sources as well as cases of unsealed sources of high activity.

Release of materials from installations that are decommissioned, like research reactors and particle accelerators. The release problems relevant to the first case is similar, from the conceptual point of view to that of the decommissioning of a power stations, although the problems are of much less quantitative relevance. The second type of problem, the decommissioning of an accelerator falls into the category of release of parts and structures that have been activated during the accelerator life.

Induced radioactivity creates release problems in connection with the research on the radiation hardness characteristics of parts, components and structures that are carried out in the framework of research in material science, space applications and R\&D activities oriented to particle physics.

4-4 Release of radioactive materials from industrial facilities 
The problems of release of radioactive materials from industrial facilities, belong to the following categories.

Release of radioisotopes that are employed in the current activity of the facility. As pointed out in the introduction, radioactive sources, in several cases featuring a high level of activity are employed in several industrial processes, like food sterilization.

Release of radioisotopes that are part of instruments employed in the facility. This is the case of thickness and level meters based upon radioactive sources or of static eliminators utilizing the ionization produced by a radioisotope.

Release of final products that include a radioactive source, like for instance a smoke detector.

Release of final products that may have become active as a result of the particular processing they have been submitted to. An example of this situation provided in the introduction is the irradiation of gems, like topaz, which remain radioactive after neutron irradiation.

Release of final products that may have become active as a result of the reprocessing of materials where a radioactive source was present and remained undetected. Examples of this situation have occurred in steel recycling facilities, where a radioisotope was mixed in the scrap to be melted. There is a growing concern about the large-scale release of radioactive metals into the public domain, which results from the decommissioning of several nuclear installations.

\section{5-DOE-SPECIFIC RELEASE PROBLEMS}

The following sections are devoted to the release problems that are specific to DOE activities and to the decommissioning of DOE installations and facility. On the basis on the previous discussion about the general aspects of release of radioactive items, it is clear the these problems are of extreme importance for DOE, owing to the large number of radiation-related facilities and installations that are under its responsibility.

It is also clear that the importance of the problem of the release of radioactive items from DOE facilities will continue to grow as more and more radioactivity-based facilities are decommissioned and dismantled. It must be pointed out also that and more and more sophistication is required in the release process to assess the level of the risk for the operators, the population and the environment that the release of radioactive items may bring about. It is clear that radioactivity-based facilities will continue to be built and their aging process will make their dismantlement inevitable after a certain number of years. The associated release of materials and structures will continue to be an extremely important aspect in future DOE activities. The following questions need to be answered in relationship with the material release:

1 - Can a radioactive item be removed from the installation, transported and stored into a suitable site? What safety precautions are needed to accomplish these tasks?

1 - Where can a given item be released? The site where it will be eventually conveyed depends obviously on its level of activity.

2 - Is it possible to fix safety activity limits such that a given material be released into the public market? 
Question 2 has a very broad significance, as it includes the case where an entire building, which has for some time employed for radiation-related activity can be assigned to other activities and even put on the market as a private house or as a non-radiation related industrial facility..

The answer to these questions is related to a reliable assessment of the activity of the items to be released. The general discussion has shown that the items that need to be released in the decommissioning of radiation-based activities belong to different categories.

a) High-activity wastes coming from the decommissioning of nuclear reactors, weaponsproducing facilities and hot cells that require to be moved to a final storage site.

b) Highly contaminated liquids that need to be processed

c) Activated metal structures that belonged to nuclear reactors and may feature high levels of activity

d) Rubble coming from activated blocks of concrete and contaminated walls of buildings

e) Rubble, metal structures and parts featuring low to very low levels of activity.

The last point is of particular interest, because it may open-up a recycling process of the enormous amounts of valuable metals that result from the dismantlement of some of the radiation-based facilities, like a nuclear power station or a nuclear submarine.

In all the situations a) through e) considered above the radiation monitoring is a fundamental step in making the decision on the possible use or method of disposal of the radioactive material under consideration, as it will be shown in the section devoted to monitoring..

\section{5-1 Decommissioning, refurbishing or change of use of a nuclear facility}

The decommissioning, refurbishing or the change of use of a nuclear facility present several aspects of interest related to their clearance.

The first is the release of the building itself, the question being whether or not the building can be cleared for a different use or even put on the real estate market. The nature of the previous activities in the building, the intensity of the radiation sources employed in those activities and the time the installation has been in operation determine whether one of the previous options are viable or whether the building needs to be demolished.

The second one is related to the case where the building needs to be refurbished during its normal operation and the resulting rubble must be released for disposal. The rubble can come from the demolition of walls in the building or from the biological shield in nuclear reactors or from the shielding walls in accelerators.. As a general rule, the rubble presents a comparatively low activity. The activity on the walls is predominantly due to contamination by deposits, and a lesser extent, to induced radioactivity. The radioactivity due to contamination may penetrate the structure, although the penetration is limited to a depth of the order of $1 \mathrm{~cm}$. In the case of the shielding concrete blocks employed in nuclear reactors and accelerators the induced activity may be more pronounced than in the previous case. The assessment of the rubble activity is an important issue. An accurate assessment of the activity allows, indeed, the decision about the correct disposal of the rubble. If, by monitoring it, it is possible to ascertain that the activity of the rubble 
is sufficiently low to allow an ordinary disposal, that is, not subject to the strict and costly regulations governing the disposal of waste originated from radiation-related sites, this may result in a substantial reduction of the cost of disposal.

The saving can be much more substantial in the case of the demolition of the facility, where the amount of rubble is much larger. Besides, the rubble can be reused in several applications. Again, the accurate assessment of the activity of the rubble is of paramount importance, because it may suggest to which type of reuse the rubble can be directed. The rubble, in other words, depending on its level of activity can be employed in certain applications or discarded for others, where it may create hazard to the population or to the environment. The problem of assessing the activity of rubble prior to its release will be discussed in detail in one of the forthcoming sections.

\section{5-2 Release of radioactive items from weapons-producing facilities}

Huge release problems arise in connection with the downsizing and decommissioning of weapons-producing facilities. The first steps involved in the production of nuclear weapons and therefore expected to generate wastes and radioactive materials and objects are to some extent similar to those described with reference to the fuel cycle for electricity generation. They are; uranium mining, milling, refining and enrichment. They are followed by the specific weapon fabrication steps. The release problems involve highly active materials as well as low and very-low activity wastes. Contamination and activation play a very important role in the material release aspect connect with the weaponsproducing facilities.

\section{5-3 Release of radioactive items from hot cells}

The hot cells, that are intended to process radioisotopes of extremely high activity are characterized by correspondingly high levels of contamination and activation. Dismantling a hot cell facility presents very serious problems of material release. Every structure, as every part of the cell is active and their release causes very serious problems. A hot-cell facility in the Sorrento Valley area, near San Diego has been decommissioned, decontaminated and dismantled between 1995 and 1998. In the decommissioning process it was found it was found that roof, external walls and floors were contaminated, with penetration of the contaminants into their structures. Also contaminated, though to a lesser extent was the soil around.

\section{5-4 Release of metals and metallic structures}

It was already pointed out that the decommissioning of DOE managed nuclear installations may provide enormous quantities of valuable materials that, if recognized to present radioactivity below preset limit may be recycled or even be put on the market in unrestricted conditions. However, in order to do that, a very accurate assessment of the level of activity is necessary prior to making any decision in that sense. The recycled metals, indeed, may find applications that put the items obtained from recycling in close contacts with persons. The recycled metals, indeed, can be employed in highly diversified industries, from automobile to electric appliances, to reinforced concrete in the buildings. 
On the other hand, the proper use, under safe conditions, of the metals and metallic structures obtained from the decommissioning of the facilities may be economically worthwhile.

\section{6-ASSESSING THE ACTIVITY OF THE ITEMS TO BE RELEASED}

The importance of an accurate assessment of the activity of the items to be cleared for release has been pointed out exhaustively in the previous sections. The assessment is based on an extensive use of radiation monitoring by radiation detectors and detection techniques.

The discussion on the situations arising in the release of radioactive materials shows that there is a large variety of radioisotopes and activated materials emitting different types of radiation and consequently requiring different types of detectors for the assessment of their activities. The radioactivity may come from nuclear fuel and fission products $\left({ }^{90} \mathrm{Sr}\right.$, $\left.{ }^{137} \mathrm{Cs},{ }^{235} \mathrm{U},{ }^{238} \mathrm{U},{ }^{239} \mathrm{Pu}\right)$ and radionuclides created by the neutron flux $\left({ }^{55} \mathrm{Fe},{ }^{60} \mathrm{Co},{ }^{63} \mathrm{Ni}\right)$. Numerous other isotopes can also be present, especially in the hot cell case.

This section is devoted to the discussions of the solutions to be pursued in order to cover the broad variety of radiation types which are encountered in the release of radioactive items. In some cases, the mere assessment of the activity may not be sufficient and identification of the type of emission must be determined by spectrometry techniques.

The extremely broad range of activity levels that may be encountered is such to require techniques that rule out the presence of the human operator and require a monitoring done on a robotic case, like for instance in the case of the hot cells or a power reactor core to the other extreme case, where low-level counting techniques are to be employed to detect counting levels below the natural background.

As suggested in the introduction, the assessment of the activity levels in the items to be released may require an $\mathrm{R} \& \mathrm{D}$ effort in the area of detection techniques in order to solve some specific problems. This effort will be highly rewarding in terms of the risk to which the population and the environment may be exposed as well as in term of the financial gain that could arise from the reuse or recycling of the released items.

Some particular cases will now be examined in detail.

\section{6-1 Case of extremely high radiation doses. Monitoring hot cells and reactor cores}

Extremely high activities are present in the hot cells and in the cores of power nuclear reactors. The levels of activity can be so high to rule out the intervention of a human operator in the radiation monitoring process, which must rely entirely on a robotic approach. The radiation levels may be so high in some cases to impair even the operation of the detector as well as of the electronics which supervises the operation of the robot and processes the data from the detector. There is a rich literature addressing the issues of the radiation hardness in detectors and electronic circuits. A large amount of information comes from the R\&D activities associated with the detector studies in particle physics, where the experiments on high luminosity accelerators have stimulated the studies aiming at realizing detectors and circuits able to stand extremely high radiation doses. The case of the hot cells and the reactor core, however, present requirements that are different from the ones arising in particle physics. The radiation to be detected in the hot cell and in the 
reactor core cases consists of gamma rays, neutrons, $\alpha$ and $\beta$ particles. The environment is also generally much more hostile than the ones encountered in particle physics. In the actual cases there will be also chemically aggressive substances, requiring a particular ruggedness in the detector. A detector material which suits this requirement and was proven to be able to stand extremely high radiation doses is diamond. Diamond is a very good detector of $\alpha$ and $\beta$ particles. However, diamond is a very low $\mathrm{Z}$ material and as such unsuitable for the detection of $\gamma$ rays. This obstacle can be circumvented by thinking of a detecting structure based on the $\gamma$ conversion process as described in fig. 1.

CONVERSION DETECTOR

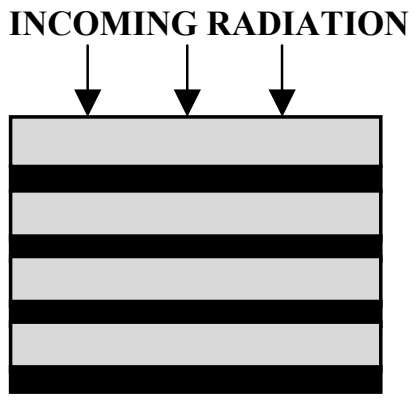

Fig. 1 - Proposed structure of a conversion-type detector with alternated layers of converting and detecting materials.

detecting material converting material

The structure of the conversion-type detector is a sandwich made of alternated layers of converter materials and detectors, fig.1. To some extent this structure resembles the one adopted in sampling-type calorimeters in particle physics. Tungsten can be a choice for the converter material. The thickness of the converter layers is now subject to the compromise of achieving a sufficiently high conversion efficiency without an excessive absorption of the created electrons. It is worth noticing, with reference to fig. 1, that the first layer encountered by the radiation emerging from the scrap is a detector. This is in order to enable the system to detect electrons in case a $\beta$ emitter is present in the scrap, without stopping as it would happen if the first layer were made of the converting material. The detecting layers are made of diamond, which suits the requirements of ruggedness and radiation hardness of this applications to the best possible extent.

Detectors of artificially made diamond can nowadays be manufactured with active areas of up to several tens of $\mathrm{cm}^{2}$. The insulator nature of diamond permits a detector realization in the form of a solid state ionization chambers, made by evaporating the metal electrodes on the opposite faces of a diamond wafer .

The quality of industrial diamond for use as solid-state detectors has been continuously improving during the past few years. The parameter which is employed to characterize the quality of diamond in detector applications is the path $\lambda$ traveled by an electron from the point where it is created by the ionizing radiation to the point where it is trapped. Throughout this path the electron induces charge on the collecting electrode. Recent diamond detectors feature values of $\lambda$ in excess of $200 \mu \mathrm{m}$. The deposition of a layer of 
hydrogenated material, like a foil of plastic material, on top of the first detecting layer can extend the detecting capability of the device to the case on neutrons. (5) (6).

The problem of the radiation hard robot supervising and detector signal processing electronics can be efficiently solved by using a design based on silicon Junction FieldEffect Transistors (JFET). The junction field-effect transistors lend themselves to linear, nonlinear and switching analog applications. The feasibility of logic circuits entirely based upon junction field-effect transistors has also been demonstrated. Monolithic technologies based upon junction field-effect transistors are available. Radiation hardness tests done on monolithic circuits obtained with these technologies have shown that the circuits are still functional with a tolerable degradation in their performances after exposure to gamma-ray doses of up to $100 \mathrm{MRad}$ and neutron fluences of $6 \times 10^{14} \mathrm{~cm}^{-2}$. It can therefore be concluded that the problem of monitoring high activity sites, like the hot cells and the core of a power nuclear reactor can be solved by associating a diamond conversion-type detector with electronic circuits based entirely on junction field-effect transistors (7).

\section{6-2 General monitoring considerations for the intermediate activity case}

The following considerations refer to the case of a very preliminary assessment of the activity in sections of a radiation-related facility where the level of activity is foreseeable with a margin of uncertainty such to allow the access to operators without exposing them to any significant risk.

Should any doubt exist about this point, it advisable to begin with a roboticsimplemented monitoring approach. This, in general, will not require the level of ruggedness and radiation hardness demanded by the monitoring of a hot cell, for the levels of activation and radioactive contamination are expected to be much lower, Once the access to the human operator is cleared, monitoring will be done bearing in mind the following considerations.

1-There is a preliminary, coarse step, which consists in determining the activity on the basis of the counting rate measured by a simple Geiger-Mueller counter.

2-The Geiger-Mueller counter, however, does not provide any information about the nature of the radiation and its energy.

3-To identify the nature of the radiation and its energy, a more sophisticated counting system is advisable. This may consist of two energy-dispersive analysis systems, employing different radiation detectors.. One should be based upon a high-purity Ge detector with a mechanical cryocooler to provide high resolution analysis of $\mathrm{X}$ and gamma radiation. This system permits the identification of the radioisotopes from their characterisic spectral lines. The second system should be based on a silicon detector aiming at performing energy-dispersive analysis on $\alpha$ and $\beta$ particles.

4-A thin polyethylene layer on the radiation entrance side of the silicon detector will add to the system neutron detection capability. 
5-The problem of neutron detection may require that further options be considered, among which the boron-trifluoride proportional counter.

\section{6-3 Assessment of very low activities by low-level counting techniques}

This is probably the most interesting case because it is the process which may give clearance to the release of materials to be reused in or recycled for the applications that are most critical from the standpoint of radiation safety.

Needless to say that these are also the applications that may create the largest profit associated with the release.

The assessment of activities that may require the intervention of low-level counting techniques is usually initiated when the general monitoring procedures have been completed and it has been ascertained that the activities do not significantly exceed the natural background levels. The natural background activity can be monitored in the vicinity of the facility and outside it. Samples taken from the facility can be taken outside, their activity monitored and the natural background activity measured at some distance from the sample.

If all these tests confirm that the sample activity is comparable to that of the natural background, the low-level counting techniques can be applied. These techniques are designed to assess radiation activities that are small than the natural background.

It may be wondered why is it necessary to assess activities below the natural background. The answer is that the release standards for items coming from radiation-related facilities and intended for reuse or recycling directed to some applications feature levels of activity below the natural background.

A low-level counting system is intended to measure the activity of a sample by putting the sample to be monitored and the detector, which is called in what follows the main detector into an enclosure which shields it from the background radiation. There may be one or more main detectors in the enclosure, for the low-level counting system should have the same detection capabilities for different types of radiation as discussed in the section relevant to the general monitoring case In the design of a low-level counting system, a fundamental issue is the shield. Some types of low-level counting systems employ what is called a passive shield, that is, sample and detector are located in a chamber whose walls are made of a high $\mathrm{Z}$ material, normally lead. More effective rejection of the natural background is achieved by the use of the so called active shield, which consists of an additional detector system surrounding the sample and the main detector (8). The active shield is often referred to as a veto counter, for whenever a signal is detected in the active shield, a logic decision prevents the signal from the main detector from being acquired or counted. An example of low-level counting system employing an active shield is shown in fig.2, which represents a vertical section of the actual system. The space between BOX 1 and BOX 2, which is filled with a liquid scintillator loaded with lead in order to increase the efficiency in the detection of gamma rays, is the active shield. The scintillation light from the liquid medium in the active shield is detected by photomultiplier tubes, labeled PMT1, PMT2, PMT3, PMT4 in fig. 2. The signals from the four tubes are combined in the OR circuit. If any of them delivers a pulse, a logic signals appears at the output of the OR circuit. . The signal from the main detector is 


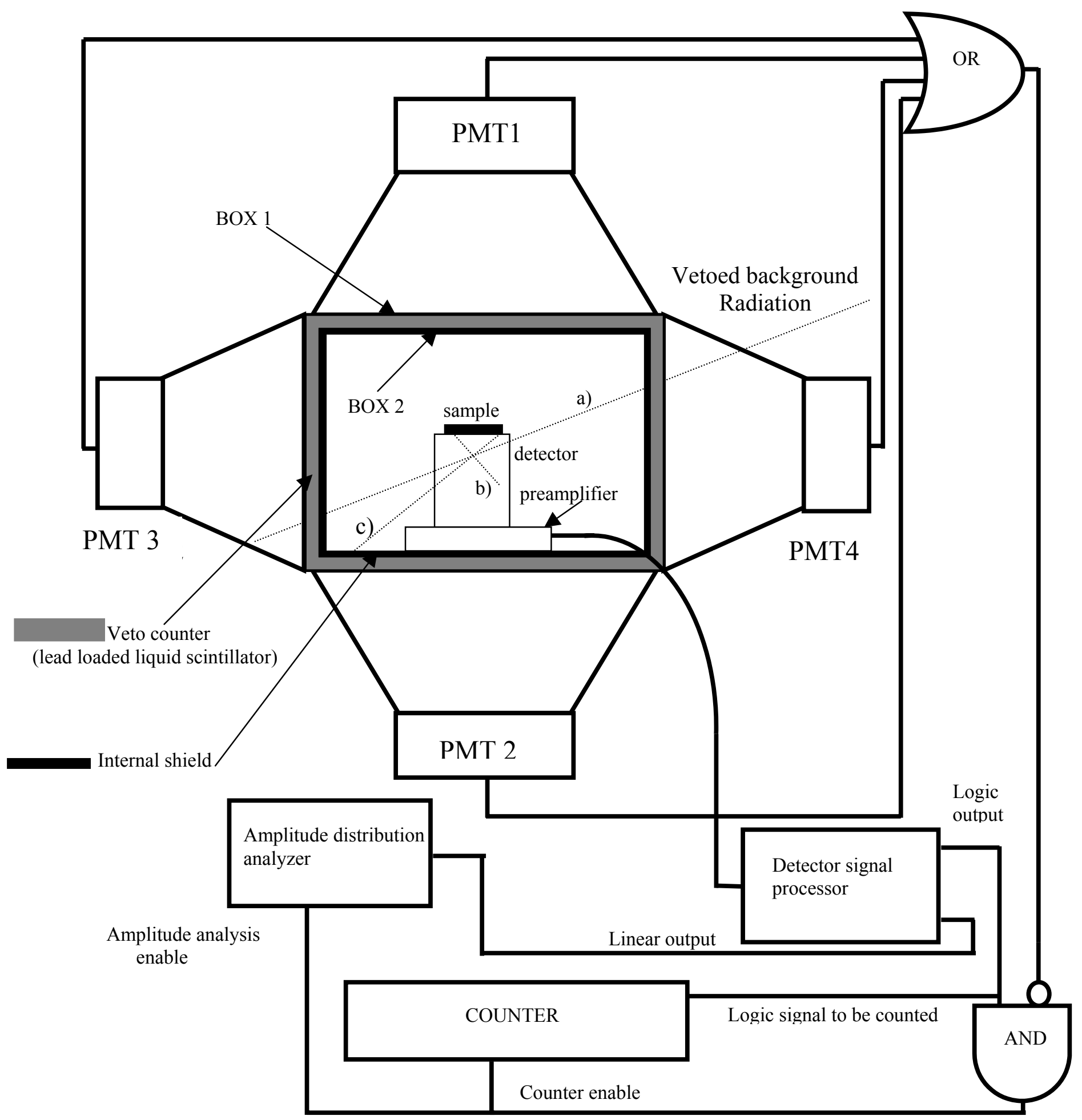

Fig. 2 - Low-level counting system based on active shield (veto counter) 
transmitted to the signal processor. This contain an analog section consisting basically of a shaping amplifier, which provides the linear output in fig. 2 . The signal from the shaping amplifier is sensed by a threshold comparator, internal to the detector signal processor. The comparator provides the logic output. The logic output is combined in the AND gate of fig. 2. with the logically inverted signal from the photomultipliers' OR. The logic output from the detector signal processor serves the purpose of counting the number of events from the sample monitored by the detector. This provides the information about the activity of the sample. The linear output from the detector signal processor is sent to an amplitude analyzer which builds up the amplitude distribution of the analog signals, thereby implementing the energy dispersive analysis. Both counting operation and amplitude analysis are enabled by the output of the AND circuit. For instance, a high .energy particle from cosmic radiation which crosses the veto counter and also the internal detector would produce a signal at the output of the OR circuit and a logic output from the detector signal processor. No enable signal would appear at the AND output and therefore, neither the counting nor the amplitude analysis would be activated. Track b) in fig. 2 is that of a particle or a photon emitted by the sample, which terminates its range within its sensitive volume. If the energy released is sufficient to trigger the comparator in the detector signal processor, a signal appears at its logic output along with an a signal at the linear output. No signal is present at the output of the OR circuit, because none of the photomultipliers was activated. A signal appears, accordingly, at the output of the AND circuit, which enables both the counting and the amplitude analysis. Case c) is that of a radiation emitted by the sample, which is detected by the i main detector, but escapes from it. If it were able to reach the veto counter, it would be lost. To prevent the occurrence of such an event, the walls of BOX 2 are covered by a layer or high $\mathrm{Z}$ material, the internal shield in fig.2, acting as an absorber. In a more elaborate approach these cases could be dealt with by segmenting the active shield and using trackingreconstruction algorithms.

As already pointed out, the main detector in the system of fig. 2 is to be chosen according to the criteria discussed for the general situation in section 6-2.

The residual contamination from the natural background in a low-level counting system employing an active shield is due to the following causes:

1. Incompletely shielded external radiation. The incomplete shielding may be due to the inefficiency of the vetoing detector as well as to the imperfections in the mutual time relationship in the signals at the input of the NAND gate.

2 Intrinsic contamination in the main detector and in the materials inside BOX2

However, the lowest detectable activities in a system of the type of fig. 2 are surely at least one order of magnitude below the natural background.

\section{7-APPLICATIONS OF LOW-LEVEL COUNTING TECHNIQUES IN THE RELEASE OF ACTIVE ITEMS}


Here are two examples of how the low-level counting techniques may intervene in the assessment of the activity of materials to be released.

7-1 Monitoring the rubble from the decommissioning, refurbishing or change of use of a nuclear facility

The rubble lends itself to an accurate monitoring of its activity. It can be crushed into pieces and these can be analyzed by field-portable low-level counting systems. The lowlevel counting system will be used in the final step of sorting the material in order to judge whether or not it can be considered for applications that are most demanding from the point of view of radiation safety. The assessment of the level of activity in the rubble should be done according the following procedure (10).

1 - A preliminary assessment is done on the walls while they are still standing, by using different types of detectors. A Germanium detector which permits a high resolution energy-dispersive analysis of the radiation is advisable, to determine the gamma-rayrelated activity. A silicon detector is useful to determine any $\beta$-emitter contamination. Monitoring should be completed by a neutron-sensitive detector, for instance a BF3 proportional counter.

2 - A careful removal of a thin layer of the wall surface, which is then counted by a $\mathrm{Si}$ detector should be employed to determine the presence of $\alpha$-particle contamination.

If the previous two tests reveal radiation levels that are so low to suggest that the rubble may be a good candidate for applications demanding a very low activity, the next step should be the low-level counting.. The activity measurement should be done in two steps:

3 - On a thin sample removed (scratched) from the wall surface

$4-$ On a piece of $1 \mathrm{~cm}$ thickness taken from the wall

The purpose of the latter measurement is to check the extent to which the activity has penetrated the structure of the wall. The low-level counting system should have the same detection capabilities for different types of radiation as in the preliminary measurements. In particular, energy-dispersive analysis may be required to determine the nature of the radiation. For this purpose, the use of a well-type Ge detector to monitor the gammaemitter contamination in samples of type 3 ) and 4).

\section{7-2 Release of metals and metallic structures from radiation-related installations}

It was already pointed out that the release of metals and metallic structures from radiation connected installations and facilities may result in significant profits provided that the activity of the items to be cleared is assessed down to levels that may be very low. Again, the low level counting techniques may play a fundamental role in the final sorting steps.

The recycling of certain metals is an important industrial issue and implies several steps that may result in a significant risk for the human operators and for the environment if the 
release is not accompanied by the safety measures that only an accurate assessment of the activity can suggest. To a much larger extent than recycled rubble, the recycled materials can end up into items that are very close to people in their daily activities. The steps in the process that leads from the metal scrap all the way to the finished product from a steel recycling plant and the related risks of exposure and environmental contamination have been exhaustively discussed in a workshop held in Dallas in 1998 and in a course in 1999. The purpose of both was giving directions aimed at improving the safety levels for the metal scrap vendors and for the operators in a steel recycling plant, as well as for people living in its proximity and for the environment. The main concern was the detection of a radioisotope hidden in the metal scrap.

He present discussion, instead, focuses on how to release metal parts that are inherently safe because their activity has been previously monitored with great accuracy. If a load of scrap of this kind is released into the steel scrap market, it can be assumed that the risk it may presents is foreseeable and controlled. However, the problem of its sorting according to the different possible applications still exists. The problem to be tackled can be formulated in the following way (11):

1-Limits are set for the maximum allowed exposure to different types of radiation for the metal scrap vendors in transporting and pre-processing it, for instance in sawing, shredding and disassembling it.

2-Limits are set for the maximum allowed exposure of workers in the steel recycling plant.

3-Limits are set for the maximum tolerable activity of the finished product in accordance with the type of application it is intended for.

These limits determine the level of activity under which the metals can be released.

Obviously, the problem is not of easy solution, because several other conditions must be considered, like the nature of the emitted radiation, which may require again high resolution energy dispersive analysis.

The activity assessment on metals and metal structures follows very much the same pattern seen for the rubble. That is, a preliminary evaluation based on robotic techniques may be requested in those cases where the activity is suspected to be very high. When it been found out that the measurement by human operator is feasible, counting and energydispersive analysis with the previously described types of detectors will be carried out. In the extreme case of very low activities, low-level counting techniques can be employed on pieces of metal, resulting, for instance, from the shredding of larger items.

\section{8-LOWEST ACTIVITIES TO BE DETECTED IN ITEMS TO BE RELEASED- THE LIMITS FOR UNRESTICTED RELEASE}

- The activity limit set by the European Union for the unrestricted (exempt) release of items from a nuclear installation or facility has been fixed in the following way. The released item must not cause individual exposure to a dose exceeding $10 \mu \mathrm{Sv} /$ year. 
Such an individual dose limit, which corresponds to an extremely strict safety condition has been fixed in compliance with a recommendation by the International Atomic Energy Commission (IAEA).

The $10 \mu \mathrm{Sv} /$ year limit to the individual exposure is extremely low, for it is consistently lower than the exposure due to natural background.

However, all those items that are to be released in compliance with the IAEA recommendation must be proven to meet this release standard. The European Union has decided to comply with the IAEA recommendation and in document "Radiation Protection 117-Methodology and models to calculate individual and collective doses from the recycling of metals from the dismantling of nuclear installations" has assumed that the $10 \mu \mathrm{Sv} /$ year individual exposure limit must not be exceeded in any of the step that intervene in the metal recycling process, that is:

- Exposure in a scrap yard

- Exposure in a foundry

- Atmospheric emission

- Exposure during post-refining process

- Exposure related to use of product

- Exposure related to the disposal or use of by-products

3 The same $10 \mu \mathrm{Sv} /$ year limit is found in European Union document "Radiation Protection 114-Definition of clearance levels for the release of radioactively contaminated buildings and building rubble"

In either case, from the $10 \mu \mathrm{Sv} / \mathrm{year}$ value assumed as an upper limit to the individual exposure, the maximum allowable activity concentration in the item suitable for unrestricted release. In the case of building or rubble release, the activity concentration is usually expressed in $\mathrm{Bq} / \mathrm{cm}^{2}$ if relevant to surface contamination or in $\mathrm{Bq} / \mathrm{cm}^{3}$ if relevant to the volume contamination or activation.

In the case of metals, the activity concentration is more adequately expressed in $\mathrm{Bq} / \mathrm{g}$.

The previous limit of the individual exposure and the resulting limit of activity concentration in the item suitable for unrestricted disposal emphasize the importance of the low-level counting techniques associated with high resolution energy dispersive analysis. The conversion coefficient from the individual dose limit to the limit in the activity concentration of the sample depends, indeed on the nature of the emitted radiation.

For instance, ${ }^{137} \mathrm{Cs}$ contamination on the walls, would require, for the reuse of a building a surface activity concentration of $1.5 \mathrm{~Bq} / \mathrm{cm}^{2}$, that is, on average 1.5 photons $/ \mathrm{cm}^{2} \mathrm{~s}$ emitted from the sample. The sample, even in a well-type Ge detector, which would cover a large solid angle, would produce a number of counts over a specified time below that arising from the natural background.

The clearance level of steel scrap based upon the $10 \mu \mathrm{Sv} / \mathrm{year}$ individual exposure limit, would be $0.57 \mathrm{~Bq} / \mathrm{g}$.

To further stress the importance of low-level counting techniques in the unconstrained release, it is useful to remember that the US average individual exposure related to 
natural background is about $4 \mathrm{mSv} / \mathrm{ear}$. So, the $10 \mu \mathrm{Sv} / \mathrm{year}$ is between two and three orders of magnitude below the exposure due to natural background.

\section{9-CONCLUSIONS}

The report has considered the problems that arise in the release of items from facilities and installations where activities based upon radiation have been carried out. For the sake of completeness, the different release problems that arise in the vast variety of radiationrelated contexts has been reviewed. Particular attention has been conveyed on the problem of monitoring the items to be released in order to assess their level of activity and sort them for the reuse, recycling and disposal. Monitoring may require very advanced detection and radiation analysis techniques in order to comply with the most stringent requirements for unrestricted release.

\section{0-REFERENCES}

1 P.F. Manfredi - Detection of radioactive sources in the metal scrap: basic principles and inherent limitations.

Workshop on the Detection of Radioactive Sources in Metal Scrap.

Dallas (Tx) 8-9 June 1998

2 -J.G. Bellian et al -Evolution of radioactive scrap monitoring systems.

Workshop on the Detection of Radioactive Sources in Metal Scrap. Dallas (Tx)

8-9 June 1998

4 B.D. Geelhood et al - Detection and measurement experience at Pacific Northwest Laboratory for monitoring of radionuclide contamination of materials and the environment.

Workshop on the Detection of Radioactive Sources in Metal Scrap. Dallas (Tx) 8-9 June 1998

5 A. La Mastra - Current detection capabilities of scrap monitoring systems Workshop on the Detection of Radioactive Sources in Metal Scrap.

Dallas (Tx) 8-9 June 1998

6 M.W.Kramer, - P.F.Manfredi et al - Status of diamond particle detectors

Nuclear Instruments and Methods - A-418 (1998) 196

7 D. Meier, P.F.Manfredi et al - Proton irradiation of CDV diamond detectors for high luminosity experiments at LHC

Nuclear Instruments and Methods - A-426 (1999) 173

8 P.F. Manfredi et al - Noise degradation induced by gamma-rays on P-and N-channel junction field-effect transistors

IEEE Trans. Nuclear Science NS-46 (1999) 1294

9 G. Heusser - The background components of germanium low-level spectrometer Nuclear Instruments and Methods B-17 (1986) 418

10 Radiation Protection 114-Definition of clearance levels for the release of radioactively contaminated buildings and building rubble

European Commission, 5 May 1999 
P. F. Manfredi - J. E. Millaud - Lawrence Berkeley National Laboratory

11 Radiation Protection 117-Methodology and models to calculate individual and collective doses from the recycling of metals from the dismantling of nuclear installations

European Commission, 2000 